www. revis tad y o. com

\title{
Análisis de la alineación de las tecnologías de la información y el negocio en empresas de la Comunidad Valenciana
}

\author{
Business and IT alignment in companies of Valencian Community in Spain
}

\author{
Llanos Cuenca, Juan de Dios Milla, Andrés Boza \\ Escuela Técnica Superior de Ingeniería Informática. Centro de Investigación Gestión e Ingeniería de Producción (CIGIP). Universitat Politèc- \\ nica de València. (UPV). Spain
}

Fecha de recepción: 21-10-2014

Fecha de aceptación: 20-02-2015

Resumen: Con el fin de generar valor para la organización, los proyectos de tecnología de información (TI) necesitan estar en consonancia con las necesidades de la organización. La gestión y mejora de esta conexión es la alineación de negocio y TI. El objetivo es analizar el grado de alineación en empresas de la Comunidad Valenciana. El análisis se ha llevado a cabo sobre una muestra de veinticuatro empresas bajo los aspectos: valor de TI, comunicación, prioridad de proyectos TI y rendimiento, innovación, fiabilidad y oportunidades de TI en la empresa. Los sectores analizados han sido actividades inmobiliarias, profesionales y administrativas.

Palabras clave: Valor de TI, Alineación de negocio y tecnología de la información, madurez, sectores empresariales

\begin{abstract}
In order to create value for the organization, the projects of information technology (IT) need to be in line with the needs of the organization. The management and improvement of this connection is the alignment of business and IT. The aim of this paper is to analyze the degree of alignment in companies of the Valencian community in Spain. The analysis was conducted on a sample of twenty-four companies under the aspects: 1) the value that the company gives the IT: The purpose of these in the company as well as the contribution made and the vision that the company has on them. It will also consider whether the company provides adequate resources to IT. 2) Communication and working relationship between the department and the company, valuing the involvement of executive management with the IT department, frequency of contact between the two departments, the degree of involvement of IT in strategic business of the company and the contribution of executive management in IT planning. Also observe this variable direction communication between the IT department and the company, how is this communication (formal or informal), 3) Knowledge between the company's business and IT: measuring the knowledge of IT on the business and the knowledge that executive management has on IT projects; It also analyzes whether there are courses or some other method to increase staff knowledge of IT on the business of the company, taking into account this knowledge when hiring IT staff, if the department knows the business objectives, 4) The priority and involvement that gives the company IT projects: measuring whether IT projects are re-flected in business plans. 5) The performance, innovation, reliability and opportunities of IT in the enterprise: to measure as it is considered the company against its competitors technologically if the IT staff is motivated to innovate in the business, if it meets the IT department with targets set by the company or trust that the department has in its ability and utility and to the company. The sectors analyzed are estate, professional and administrative activities. The data obtained in this study shows that SMEs understand IT as a way to increase the productivity of the company and to reduce costs. But, they should also be incorporated as a process, used as a competitive advantage and increase the degree of involvement to improve alignment. Communication between the two areas should be improved in most companies, it can be concluded that it is in the understanding of this paper where the problem lies not achieve the full potential of information technology. There are very few companies that feel that the business area is IT skills, and the same goes the other way. Today, companies must improve the prioritization of technology projects, which is reflected in the business plans of the company. IT meets the objectives, but there are few companies that are considered as technology leaders. Greater involvement, increased communication and greater knowledge could enhance the use of information technology more effectively, improving the alignment between both areas.
\end{abstract}

Keywords: IT Value, Business and IT alignment, maturity, company sectors 


\section{Introducción}

La tecnología de la información (TI) ha pasado a desempeñar un papel crucial en el apoyo, la sostenibilidad y el crecimiento de las organizaciones. Las organizaciones que han alineado con éxito sus negocios y la gestión de TI obtienen mejores resultados que las que no lo hacen (Henderson y Venkatraman, 1993; Chan y Reich, 2007a, 2007b; Cuenca et al, 2011; Trienekens et al., 2013). La alineación de negocio y TI es el grado en que la estrategia de TI permite e impulsa la estrategia de negocio (Cuenca et al., 2011). Entre los beneficios de una buena alineación de negocio y TI están: 1) maximizar el valor de retorno de las inversiones en TI, 2) mejorar la posición competitiva a través de los sistemas de información, 3) proporcionar dirección y flexibilidad para reaccionar a los cambios (Avison et al 2004; Haes y Grembergen, 2008). A pesar de que la alineación, ha demostrado ser muy importante, muchas organizaciones siguen tratando de abordar este problema sin éxito. Desde hace varios años, la alineación de negocios y de TI es considerada por los ejecutivos de Tl como uno de sus temas clave (Luftman et al., 2008, 2009). Parte de los resultados fueron presentados al congreso ClO2014. (CIO, 2014)

El artículo está estructurado de la siguiente manera, después de la introducción, el apartado dos detalla la metodología seguida para la selección de la muestra, las variables utilizadas, el diseño y trabajo de campo. El apartado tres expone los resultados obtenidos terminando con las conclusiones en el aparatado cuatro y las referencias utilizadas.

\section{Metodología}

Este trabajo se centra en el análisis de la situación actual de alineación entre las tecnologías de la información y el negocio en pymes de tres sectores de la Comunidad Valenciana. En concreto, la población en este trabajo son las pymes (pequeñas y medianas empresas) de la Comunidad Valenciana de los tres sectores, denominados según la Clasificación Nacional de Actividades Económicas (CNAE) de la siguiente manera (Reg, 2006): Actividades Inmobiliarias, Actividades Profesionales y Actividades Administrativas. Según datos del Instituto Valenciano de la Exportación (IVEX) (IVEX, 2012), que a su vez, se basa en los del Instituto Nacional de Estadística (INE); en la Comunidad Valenciana hay un total de 14.869 pymes en el sector de Actividades Inmobiliarias, 37.493 pymes en el sector de actividades profesionales y 12.824 pymes en el sector de actividades administrativas, lo que hace una población total a estudiar de 65.186 pymes. De acuer- do con los datos del Instituto Nacional de Estadística (INE) (Min, 2012), en Enero de 2011 el total de empresas en la Comunidad Valenciana es de 348.700 , de las cuales 348.391 son pymes, lo que supone el 99,91 $\%$ del total de las empresas de la comunidad autónoma. De las 348.391 pymes, este trabajo se enmarca en 65.186 pymes que forman parte de los sectores nombrados anteriormente. Las pymes a estudiar suponen un $19 \%$ de las pymes de la Comunidad Valenciana.

\subsection{Muestra}

La muestra o subconjunto de pymes que puedan representar a la población total la obtendremos a partir de la siguiente fórmula ya que conocemos el tamaño de la población (Cohen, 1988):

$$
\mathrm{n}=\frac{\mathrm{N} \times \mathrm{Z}^{2} \times \mathrm{p} \times \mathrm{q}}{\mathrm{e}^{2} \times(\mathrm{N}-1)+\mathrm{Z}^{2} \times \mathrm{p} \times \mathrm{q}}
$$

Siendo $\mathrm{n}$ el tamaño de la muestra que queremos conocer [1]. $\mathrm{N}$ el tamaño conocido de la población a estudiar, en este caso 65.186. El error muestral lo representa e, y admitiremos un margen de error del $15 \%, e=0.15$. $Z$ corresponde al nivel de confianza, el cuál será de un $85 \%$, que corresponde a $Z=1.44$ sigmas o errores típicos. La varianza de la población es representada con $\mathrm{p}$ y $\mathrm{q}, \mathrm{y}$ considerando que la varianza mayor (la mayor diversidad de respuestas) se da cuando $p=q=0.50$ la fórmula es siempre igual a $0.50 * 0.50=0.25$ (es una constante). Se ha considerado un nivel de confianza del $85 \%$ y un margen de error del $15 \%$ para ajustarnos a las posibilidades reales de obtener datos. En un principio se trató de obtener una muestra con un nivel de confianza mayor y un margen de error menor pero la muestra resultado era superior a las respuestas que se podían obtener, ya que la participación de la empresas era escasa, por lo que se disminuyó el nivel de confianza y aumentó el margen de error, siendo conscientes de que ello conllevaba disminuir la precisión del estudio. Aplicando la fórmula, da como resultado una muestra de 24 pymes. Estas serán seleccionadas de manera aleatoria entre nuestra población.

\subsection{Variables}

Basándonos en las propuestas de modelo de madurez de Luftman (2000) y Trienekens et al. (2013) agrupamos las variables en 5 apartados: 
EI valor que da la empresa a las TI: En primer lugar, analizaremos el valor que da la empresa a las TI, el objetivo de éstas en la empresa, así como la aportación que realizan y la visión que tiene la empresa sobre ellas. También se analizará si la empresa proporciona los recursos adecuados al departamento de $\mathrm{TI}$.

La comunicación y la relación de trabajo entre el departamento de TI y la empresa: La comunicación y la relación de trabajo entre el departamento de TI y la empresa, será medida mediante variables, en escalas de 1 a 5, valorando la implicación de la dirección ejecutiva con el departamento de TI, la frecuencia de contacto entre ambos departamentos, la comunicación existente entre el departamento de $\mathrm{TI}$ y el resto de la empresa, el grado de implicación de las TI en la planificación estratégica de negocio de la empresa y la contribución de la dirección ejecutiva en la planificación de las TI. Observaremos también con esta variable la dirección de la comunicación entre el departamento de TI y la empresa, cómo es esta comunicación (formal o informal), y si existe personal de enlace entre el departamento de TI y la empresa, y en ese caso, cuál es su aportación.

El conocimiento que existe entre el negocio de la empresa y las TI: Otro apartado de medición será el conocimiento que existe entre el negocio de la empresa y las $\mathrm{TI}$, midiendo el conocimiento del departamento de TI sobre el negocio de la empresa y el conocimiento que la dirección ejecutiva tiene sobre los proyectos de TI; se analiza también si existen cursos o algún otro método para aumentar el conocimiento del personal del TI sobre el negocio de la empresa, si se tienen en cuenta estos conocimientos a la hora de contratar el personal de $\mathrm{TI}$, si el departamento de TI conoce los objetivos de negocio, si están los planes de negocio disponible en el departamento de TI, si existen también cursos para incrementar el conocimiento del personal de la empresa sobre las $\mathrm{TI}$ y si estos se tienen en cuenta a la hora de contratar personal de dirección de la empresa.

La prioridad e implicación que da la empresa a los proyectos de TI: También se someterá a estudio la prioridad e implicación que da la empresa a los proyectos de TI, midiendo si los proyectos TI se ven reflejados en los planes de negocio, como de prioritarios son dentro de la empresa y en qué medida se incorporan nuevas tecnologías a la empresa.

El rendimiento, la innovación, fiabilidad y oportunidades de las TI en la empresa: Por último se observará el rendimiento, la innovación, fiabilidad y oportunidades de las $\mathrm{TI}$ en la empresa, para medir como se considera la empresa frente a sus competidores tecnológicamente, si el personal de TI está motivado para innovar en la empresa, si cumple el departamento de $\mathrm{TI}$ con los objetivos marcados por la empresa o la confianza que el departamento tiene en su capacidad y utilidad con y para la empresa. Se analizará también si el personal de TI ocupa más posiciones en la empresa además de ésta y si existen incentivos y su eficacia para el personal de $\mathrm{TI}$.

\subsection{Diseño}

Para realizar el cuestionario, se siguieron varias fases, desde la definición de objetivos hasta el diseño final del mismo. Teniendo como punto de partida el planteamiento del problema de manera clara y unívoca, se definieron los objetivos más específicos. A partir de los objetivos se derivan los indicadores que conforman el cuestionario, además de las categorías de respuesta de las preguntas cerradas. Las preguntas se diseñaron basadas en el documento de van Hout (2012), al haber sido este aplicado y validado en otros sectores.

Una vez realizado el diseño inicial, se llevó un pretest, encuestando a 2 o 3 candidatos para revisar las preguntas del cuestionario de tal manera que se pudiera asegurar de que las preguntas recogían toda la variación, que el significado de todos los términos era correcto, poder conocer la dificultad que tenía para los encuestados la realización del mismo y conocer el interés y la atención de los entrevistados hacia las preguntas. Mediante el pretest se revisó el contenido, la redacción y la ubicación de las preguntas, y una vez obtenidas las sugerencias de los encuestados mediante el pretest, se modificó aquello que se consideró necesario para realizar el diseño final del cuestionario que fue el que se realizó a toda la muestra. El cuestionario final constaba de 29 preguntas distribuidas de la siguiente forma, las preguntas 1 a 4 miden el valor que da la empresa a las TI. Las preguntas 5 a 12 pertenecen a la dimensión que mide la comunicación y la relación de trabajo entre el departamento de TI y la empresa. Las preguntas 13 a 20 corresponden al conocimiento que existe entre el negocio de la empresa y las TI. Las preguntas 21 a 23 son utilizadas para medir la prioridad e implicación que da la empresa a los proyectos de TI. Por último las preguntas 24 a 29 corresponden al rendimiento, la innovación, fiabilidad y oportunidades TI en la empresa. El trabajo de campo para la toma y recolección de encuestas, se desarrolló durante el periodo Mayo-Julio del año 2013. Este proceso se trabajó tanto de manera presencial como online. Una vez aplicada la encuesta, y comprobando que ya se contaba con todos los datos requeridos, se comenzó el proceso de comprobación y validación de datos para su posterior análisis y obtención de resultados. Este proceso 
se hizo separando las preguntas a partir de las variables antes mencionadas, acumulando los datos para su posterior recuento y análisis. Se cruzó también con el tamaño de la empresa. Así su análisis se realizó obteniendo los porcentajes de respuestas y destacando aquellos casos en los que la tasa de respuesta aumentara o disminuyera considerablemente según el tamaño de la pyme.

A continuación se describen los resultados obtenidos.

\section{Análisis de Resultados}

El análisis de la alineación de las tecnologías de la información con el negocio de las pymes lo proyectamos en torno a los ejes correspondientes a las variables identificadas anteriormente.

Valor de las TI en la empresa: En la mayoría de los casos las pymes tienen como objetivo principal del área de TI el aumentar la productividad (96\%) (Figura 1). Reducir costes también es uno de los objetivos que las empresas ven propio de las TI, pues hasta un $83 \%$ de las consultadas lo indican. Sin embargo, las empresas no parecen valorar a las $\mathrm{TI}$ como un proceso más de negocio, pues sólo un $13 \%$ de las encuestadas marcan esto como un objetivo. Un $29 \%$ valoran las $\mathrm{TI}$ para dirigir procesos, es observable que de ese $29 \%$ sólo un 4\% son empresas de menos de 50 empleados, siendo el $25 \%$ restante empresas más grandes. Por último un $58 \%$ entienden a las TI como la forma de tener una ventaja frente a sus competidores.

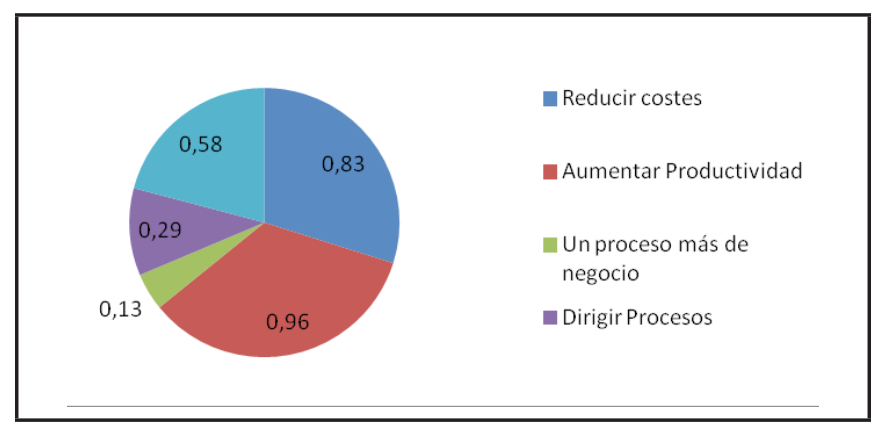

Figura 1. Objetivo principal de la TI en la empresa.

En cuanto a aportación, la totalidad de las empresas encuestadas marcan las TI como un activo de la empresa que permite actividades de negocio. Un 50\% ven las $\mathrm{TI}$ como una forma de calcular los costes del negocio, mientras que sólo un $21 \%$ las ve capaces de dirigir actividades negocio. Todas las que valoran las TI de esta manera marcaban como uno de sus objetivos principales, dirigir procesos de negocio, demostrando de esta manera que sí las ven capaces de dirigir actividades de negocio. Sólo un $13 \%$ marcan la capacidad de las TI a la hora de asociarse con otras empresas como una aportación de éstas. La dirección de las empresas consultadas ven el papel de las TI en la misma como una visión a largo plazo en el $79 \%$ de los casos, en un $92 \%$ la valoran a corto plazo, formando parte de ellas el $79 \%$ de los que las valoran también a largo plazo. Sólo un $8 \%$ no tienen una visión concreta sobre la misma. El total de las empresas está medianamente de acuerdo en que se aporta recursos adecuados al departamento de $\mathrm{TI}$, pero sólo un $17 \%$ está totalmente de acuerdo.

Comunicación y relación de trabajo: Las empresas encuestadas con menos de 10 empleados no cuentan con un departamento específico para tecnologías de la información y dependen siempre de otro departamento, lo que parece conllevar a que la implicación de la dirección ejecutiva siempre es muy alta. En las empresas con mayor número de empleados y con departamento específico, si consideran que hay una buena implicación aunque no tan alta como las anteriores. En un $92 \%$ de los consultados, se considera que hay un contacto de 3 o mayor dentro de una escala de 1 a 5 , siendo 1 nunca y 5 mucho. Dentro de ese $92 \%$, en el $33 \%$ de los casos, se considera que estos contactos se producen con mucha frecuencia. También coinciden un $92 \%$ de los encuestados en que hay una comunicación de 3 o más (siguiendo la misma escala que antes) entre el departamento de TI y la dirección. Hay una tendencia, de ser más formal, cuanto más grande es la empresa, y más informal cuanto más pequeña. Esta relación de tamaño de la empresa también afecta a la hora de tener personal de enlace entre el departamento del TI y la empresa, ninguna empresa de menos de 10 empleados cuenta con ese personal, aunque entre todas las empresas encuestadas sólo un 38\% de las pymes cuenta con este personal, y en todos los casos vienen a resumir su aportación como "interlocución entre ambas partes" (Figura 2).

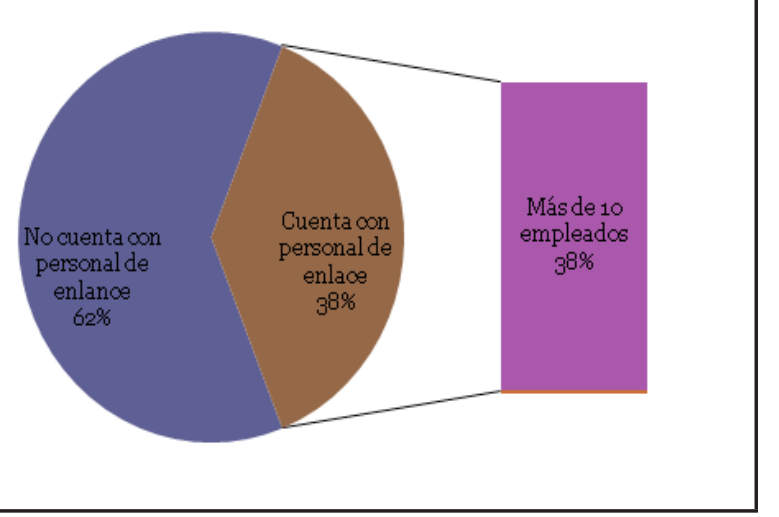

Figura 2. Personal de enlace entre el departamento de TI y la empresa. 
Es en el grado de implicación de las TI en la planificación del negocio, y el de la dirección ejecutiva en la planificación de las TI donde el porcentaje es más pequeño, pues sólo un $17 \%$ consideran un grado de implicación máximo a la hora de la participación de las TI en la planificación del negocio y un $28 \%$ a la hora de que sea la dirección ejecutiva la que se implique en la planificación de las TI.

Conocimiento entre la empresa y las TI: Sólo una cuarta parte de los encuestados afirman que el departamento del $\mathrm{Tl}$ tiene un máximo conocimiento sobre el negocio de la empresa. En un $92 \%$ de los casos no se tienen en cuenta conocimientos de negocio de la empresa para contratar personal de TI (Figura 3). Aunque sí se afirma en un $79 \%$ de los consultados que el personal de $\mathrm{TI}$ conoce los objetivos de negocio de la empresa y un $75 \%$ cuenta con los planes del negocio disponibles para el departamento de TI. En un $21 \%$ de las empresas encuestadas cuentan con cursos u otros métodos para que el personal de TI aumente el conocimiento sobre el negocio de la empresa, aunque en todos los casos son voluntarios y además todos son en empresas con más de 50 empleados. Sin embargo no hay cursos en las empresas encuestadas para que el personal de la empresa aprenda conocimientos sobre las $\mathrm{TI}$, y sólo en un $13 \%$ se tiene en cuenta conocimientos sobre las $\mathrm{TI}$ a la hora de contratar personal de dirección.

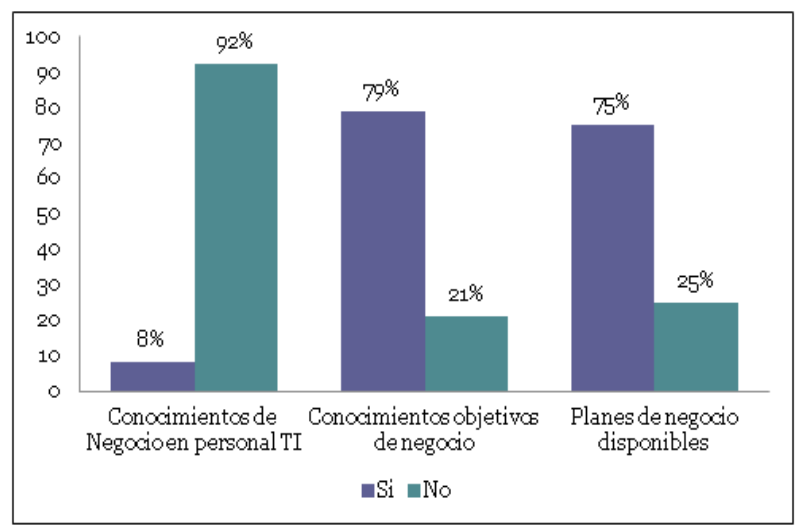

Figura 3. Conocimiento entre la empresa y las TI.

En la mayoría de los departamentos del TI se cuenta con los planes y objetivos del negocio de la empresa, pero en casi ninguna se tienen en cuenta conocimientos del negocio a la hora de contratar personal de TI, y en muy pocas se cuenta con cursos para que aumenten estos conocimientos. Ahora, al revés la implicación es aún menor, no existen cursos para aprender conocimientos sobre las $\mathrm{TI}$, y son muy pocos las casos en los que se tienen en cuenta estos conocimientos para contratar personal de dirección.

Prioridad e implicación a los proyectos TI. Ningu- na de las empresas encuestadas está totalmente de acuerdo en que los proyectos $\mathrm{TI}$ se vean reflejados en los planes de negocio. Y en ninguno de los casos se da máxima prioridad a los proyectos TI en la empresa. En cuanto a la incorporación de nuevas tecnologías a la empresa sólo un $13 \%$ afirma que sea muy frecuentemente. La prioridad e implicación de la empresa con los proyectos nunca cuenta con máxima prioridad y tampoco se ven muy reflejados en los planes de negocio.

Rendimiento, innovación, fiabilidad y oportunidades: Sólo en un 13\% se considera a la propia empresa como un líder tecnológico frente a sus competidores. Sólo un $8 \%$ creen que el personal de TI está totalmente estimulado para crear ideas innovadoras. Aunque en un $71 \%$ de los casos se considera que el departamento $\mathrm{TI}$ cumple con sus objetivos, sólo un $13 \%$ cree que el departamento de TI tiene máxima confianza en su capacidad y utilidad de sus funciones en la empresa. Como último dato sólo en un $4 \%$ existe incentivos, a los cuales se les considera eficaces. Aunque en la mayoría de los casos se considera que se cumplen los objetivos del TI con la empresa, también se observa que tanto la empresa como el departamento de TI no parece que crean que dan lo máximo de su capacidad.

\section{Conclusiones}

Los datos obtenidos en este trabajo reflejan que las pymes entienden las TI como una manera de aumentar la productividad de la empresa y así poder reducir costes. Pero, debería también incorporarse como un proceso más de negocio, utilizarse como ventaja competitiva y aumentar el grado de implicación para mejorar la alineación. Cabe observar, que las que lo hacen, tienen todas más de 100 empleados, por lo que se puede afirmar que las empresas más pequeñas no ven a las tecnologías de la información como un proceso más en el negocio de la organización, y por tanto no se le dedica la atención suficiente, lo que no les permite obtener las ventajas esperadas.

Los porcentajes en los que se afirma una frecuencia de comunicación, o un grado de implicación máximo entre la empresa y el departamento de $\mathrm{TI}$ son pequeños y nunca superan la mayoría, lo que evidencia una falta de comunicación e implicación de la empresa con las TI. La comunicación entre las dos áreas debe mejorarse en la mayoría de las empresas.

Se puede concluir que es en esta comunicación donde radica parte del problema para no poder explotar todo el potencial de las tecnologías de la información. Son 
muy pocas las empresas que consideran que el área de negocio tiene o debe tener conocimientos de las TI, y lo mismo pasa en sentido contrario. Esto implica una gran limitación en la alineación de objetivos y requerimientos, lo que repercute en la mayoría de proyectos fallidos.

Hoy en día las empresas deben mejorar la priorización de los proyectos de tecnologías de la información, lo que se ve reflejado en los planes de negocio de la empresa. Las TI cumplen los objetivos marcados, pero son pocas las empresas que se consideran como líderes tecnológicos. Una mayor implicación, una mayor comunicación y unos mayores conocimientos, podrían potenciar el uso de las tecnologías de la información de una manera más eficaz, mejorando la alineación entre ambas áreas.

\section{Agradecimientos}

Esta investigación ha sido llevada a cabo en el marco del proyecto de innovación y mejora (PIME/2013/A/016/B) 'RECICRE - Rúbrica para la evaluación de la competencia de innovación, creatividad y emprendimiento'. Avalado por la Universitat Politècnica de València y la Escuela Técnica Superior de Ingeniería Informática.

\section{Referencias}

AVISON, D., JONES, J., POWELL, P. and WILSON, D. (2004)."Using and validating the strategic alignment model". Journal of Strategic Alignment Systems, Volume 13, pp. 223 -246.

CIO 2014 CIO-ICIEOM-IIIE 2014, "8th International Conference on Industrial Engineering and Industrial Management" (XVIII Congreso de Ingeniería de Organización), "XX International Conference on Industrial Engineering and Operations Management" and "International IIE Conference 2014".

CHAN, Y. and REICH, B. H. (2007b). "IT Alignment: what have we learned?". Journal of Information Technology, 2007(22), pp. 297-315.

CHAN, Y. and REICH, B. (2007a). "IT alignment: an annotated bibliography". Journal of Information Technology, 2007(22), pp. 316-396.

COHEN J. (1988). Statistical Power Analysis for the Behavioral Sciences, second edition. Hillsdale, N.J.: Lawrence Erlbaum prise engineering approach for the alignment of business and information technology strategy". Int. J. Computer Integrated Manufacturing 24(11): 974992 (2011)

VAN HOUT E., BSC. (Febrero 2012). Measuring Strategic Business-IT Alignment. Disponible en http://alexandria.tue.nl/extra1/afstversl/wsk-i/hout2012.pdf

HAES, S. D. and GREMBERGEN, W. v., (2008). Best practices in IT governance and alignment. In: IT Service Management Global Best Practices. sl:VanHaren Publishing, pp. 47-56.

HENDERSON, J. C. and VENKATRAMAN, N., (1993). "Strategic Alignment: Leveraging information technology for transforming organizations". IBM Systems Journal, 38(2\&3), pp. 472-484.

IVEX (Instituto Valenciano de la Exportación). Generalitat Valenciana (Abril 2012): Sector Servicios de la Comunitat Valenciana.

LUFTMAN, J. and KEMPAIAH, R., (2008). "Key Issues for IT Executives 2007”. MIS Quarterly Executive, $7(2)$.

LUFTMAN, J., (2000). "Assessing Business-IT Alignment Maturity". Communications of the Association for Information Systems (AIS), 4(14).

LUFTMAN, J., KEMPAIAH, R. and Rigoni, E., (2009). "Key Issues for IT Executives 2008". MIS Quarterly Executive, 8(3).

MINISTERIO DE INDUSTRIA, ENERGÍA Y TURISMO (Abril 2012). Estadísticas Pyme, evolución e indicadores $n^{\circ} 10$.

REGLAMENTO CE 1893/2006 del Parlamento Europeo y del Consejo, de 20 de diciembre de 2006 Disp.: http://eurlex.europa.eu/LexUriServ/LexUriServ.do?uri=CELEX:32006r1893:es:

TRIENEKENS J., ROB J. KUSTERS and CUENCA L., (2013). "Measuring Business-IT Alignment, framework development and case study results", 22nd International Conference on Information Systems Development (ISD2013), Sevilla, Spain. 